\title{
User evaluation study on illumination requirements to design an augmented reality projector for open liver surgery
}

Shivaraman Ilango, Marvin Knöchelmann, Lüder Alexander Kahrs, Alexander Wolf, Tobias Ortmaier, et al.

Shivaraman Ilango, Marvin Knöchelmann, Lüder Alexander Kahrs, Alexander Wolf, Tobias Ortmaier, Roland Lachmayer, "User evaluation study on illumination requirements to design an augmented reality projector for open liver surgery," Proc. SPIE 10693, Illumination Optics V, 106930R (28 May 2018); doi: 10.1117/12.2309667

SPIE. Event: SPIE Optical Systems Design, 2018, Frankfurt, Germany 


\title{
User evaluation study on illumination requirements to design an augmented reality projector for open liver surgery
}

\author{
Shivaraman Ilango*a, Marvin Knöchelmann ${ }^{\mathrm{b}}$, Lüder Alexander Kahrs ${ }^{\mathrm{a}}$, Alexander Wolf ${ }^{\mathrm{b}}$, \\ Tobias Ortmaier ${ }^{\mathrm{a}}$, Roland Lachmayer ${ }^{\mathrm{b}}$ \\ aLeibniz Universität Hannover, Institute of Mechatronic Systems, Hannover, Germany; \\ ${ }^{\mathrm{b}}$ Leibniz Universität Hannover, Institute for Product Development, Hannover, Germany
}

\begin{abstract}
Projector based augmented reality serves as an alternate visual guidance tool for surgeons when performing complicated open surgeries. In projector based augmented reality, image overlay projection is a technique that allows the surgeon to view the underlying anatomical information such as tissues, tumors etc. directly on the surface of the organ or the patient. This will provide an intuitive view of the surgical navigation data by combining the surgeon's real world view with the preoperative three dimensional virtual models or instructions. Thus the strain on the surgeon to mentally align and visualize the preoperative data with intraoperative scene is greatly reduced. There are multiple stationary and handheld projectors available in the market today for this purpose. During surgery, stationary projectors mounted on a rack or under the ceiling suffer from a loss of adjustability and further cause shadowing issues when the surgeon occludes the scene. Although hand-held projectors do not have these disadvantages, they have major problems in terms of illuminance and luminous flux. The amount of light at which the hand-held projectors can project virtual additional information on to the patient is very low especially when the surgical lights are switched on. This paper therefore aims to provide an analysis of the requirements for designing such a special hand-held, augmented reality projector system that could be used during surgery, through a user study. Various optical parameters which are a key to design an augmented reality projector such as illuminance, luminance, luminous flux etc. are measured. Apart from that, other parameters such as refresh rate, image size, resolution which are also some important criteria in designing such a special projector, are discussed in this paper with respect to our application.
\end{abstract}

Keywords: Mixed-Reality, hand-held projector, illuminance, contrast ratio, luminance, visibility contrast, Weber Contrast, specular reflections

\section{INTRODUCTION}

Today's surgical interventions are characterized by a high degree of complexity. According to the WebMD and National practioner database report an alarming number of surgical errors are committed every year due to wrong surgical procedures. A statistical data analysis taken between 1990 and 2010 estimates that around 4000 surgical errors happen every year [1,2]. Another survey taken by the Kaiser Family Foundation reports that around 34\% of the adults from a total of 2000 adults experienced preventable medical errors [3]. This is the result of continuous increasing demands that are placed on the performing surgeon. These surgical errors may lead to post trauma complications particularly when it comes to open surgeries as these surgeries involve bigger incisions and longer healing time. Hence open surgeries have to be planned carefully. The surgeons are often provided with a complex set of medical imaging data like the Magnetic resonance imaging (MRI) or the Computed tomography (CT) data before the surgery to perform some pre-operative planning. Based on these datasets the surgeons would have to mentally visualize the location of anatomical risk structures like vessels, tissues, tumors etc. and plan or identify the incision paths in such a way that the other tissues or vessels are not affected during the surgery. Mentally aligning both the pre-operative and intra-operative scenes is sometimes a challenge and a strain to surgeons, particularly when the vessel structures are very fine or complicated. As a result, image guided systems are used sometimes, where the planned data is transferred to the operation theaters and displayed to the surgeons on the monitor screens.

*shivaraman.ilango@imes.uni-hannover.de; phone 49511762 19598; fax +49 511762 19976; https://www.imes.unihannover.de/

Illumination Optics V, edited by Tina E. Kidger, Stuart David, Proc. of SPIE Vol. 10693, 106930R · @ 2018 SPIE · CCC code: 0277-786X/18/\$18 · doi: 10.1117/12.2309667 
However, this technology requires the surgeon to divert his/her sight from the patient to the monitor screens displaying the virtual information. This becomes cumbersome for the surgeon, particularly when the surgery has to be performed for a longer duration. To circumvent these problems, alternate visual guidance methods based on augmented reality (AR) approaches are the subject of current research. To date, many AR technologies have been developed based on video overlay techniques, semitransparent displays, head mounted displays (HMD), standard video projectors etc., but they have had their own drawbacks in terms of being very intrusive, suffering from line of sight occlusions and restricting the surgeons peripheral vision $[4,5,6]$. Therefore an effective solution would be to use hand-held projectors to visualize the underlying anatomical structures directly on the surface of the skin or the organ of the patient through image overlay techniques [7]. Although projection of anatomical information and surgical guidance data onto the patient is currently the most intuitive AR solution, the amount of research carried out in this field is very less, particularly when it comes to using this technology in open surgery.

Sugimoto et al. [7] and Tardif et al. [8], among others, were the first to project anatomical models onto the patient surface using standard video projectors positioned statically above the patient. Limited by several aspects, e.g. optical system, overall size etc., commercially available video projectors cannot be easily used during surgery [4,7]. Despite these limitations, Sugimoto et al. concluded that the image overlay assisted in the 3D understanding of anatomical structures leading to significantly improved surgical outcomes resulting from reductions in operation time, intraoperative injuries, and bleeding. Gavaghan et al. developed a portable image overlay projection device (IOD) that could be integrated into the existing surgical navigation system thus, solving patient registration problems [4]. However, this device uses marker-based external tracking, which is still intrusive to the surgical environment, and the paper concluded that the light intensity of the projector (PicoP, Microvision Inc., WA) was only 10 lumens, which was clearly a limiting factor when it had to be used under surgical lights. Therefore, the operation room (OR) lights had to be dimmed while the IOD was being used [4,5]. Similarly, Edgcumbe et al. developed a mobile projector called Pico Lantern which would be inserted into the patient and picked up by a laparoscopic tool [9]. The Pico Lantern was used for surface reconstruction of the organ using structured light approach. However, Edgcumbe concluded that the projector had luminous flux as low as 15 lumens and it was a great limitation. He further added that the improvements to the technology of pico projectors would provide better accuracy, luminance and resolution in the future.

Ni et al. from Microsoft Research developed a Pico projector based doctor-patient communication device but concluded that the projector had a very low luminous flux of only 50 lumens [10]. Besharati et al. carried out an augmented reality guided neurosurgery using image projection techniques but the projection device used in this case had a very low luminous flux of 20 lumens and therefore could not be used under the surgical lights [11]. A prototype developed by Kobler et al. focused on projector based augmented reality for skull surgery but the luminous flux of the projector was only 30 lumens and this was a disadvantage [12]. Krempien et al. used projector based augmented reality for intuitive intraoperative guidance in interstitial brachytherapy. This projector had a luminous flux of around 3,000 lumens but still the surgical lights still had to be dimmed while performing the surgery [13]. Prototypes developed by DLR called the DLR 3D-Modeller [14] and VR-Map [15] use laser scanner systems to visualize the preoperative data intraoperatively. However a proper user study of these systems under the surgical lights has not been conducted. Therefore conclusive evidences cannot be inferred about the visibility of the projected data under the surgical lights.

Shi et al. built a pico projector based augmented reality display for a surgical microscope but found out that it was not suitable under surgical lights as it had only 50 lumens as optical output [16]. Glossop et al. developed a laser projector augmented reality system called XarTrax which used galvanometrically controlled mirrors to steer the laser beam. This system however had limitations in the amount and type of information that could be projected. Only 50,000 points could be generates which limits the projection of detailed information. Furthermore no hint is given whether this system was tested under the surgical lights [17]. Watts et al. used an Epson PowerLite having a luminous flux of 3,000 lumens to project medical images directly on the patient [18]. However, again there is no information given about its performance under surgical lights. Chae et al. used projector based augmented reality in angiography and concluded that the brightness of the projector was very low and therefore all the lights in operation theatre were turned off to make the image visible [19].

Previously a small user evaluation was conducted by our team on pig's liver with a projector having a luminous flux of 5,000 lumens under different surgical light intensities. At the maximum surgical light intensity, the users suggested that they were barely able to see the projected vessel structure on the liver. So the illuminance of the surgical light had to be dimmed to a lower level to visualize the projection clearly [in print]. Apart from these methods, further methods from the literature are compared and presented in Table 1. 
Table 1: State of the art review on various projection systems including our targeted system

\begin{tabular}{|c|c|}
\hline Authors & $\begin{array}{c}\text { Projector type } \\
\text { (luminous flux [lm]) }\end{array}$ \\
\hline Gavaghan et al. [4,5,6] & hand-held (10) \\
\hline Edgecumbe et al. [9] & hand-held (15) \\
\hline Ni et al. [10] & hand-held (50) \\
\hline Besharati et al. [11] & hand-held (20) \\
\hline Krempien et al. [13] & stationary (3,000) \\
\hline Schwier et al. [15] & hand-/robot-held (n/a) \\
\hline
\end{tabular}

\begin{tabular}{|c|c|}
\hline Authors & $\begin{array}{c}\text { Projector type } \\
\text { (luminous flux [lm]) }\end{array}$ \\
\hline Shi et al. [16] & hand-held (50) \\
\hline Glossop et al. [17] & stationary (n/a) \\
\hline Watts et al. [18] & stationary (3,000) \\
\hline Chae et al. [19] & hand-held (100) \\
\hline Wang et al. [20] & stationary (n/a) \\
\hline Our targeted system & hand-held (7,000- \\
& $10,000)$ \\
\hline
\end{tabular}

All the above mentioned technologies have either used markers or external tracking systems to project anatomical information. While screwing markers is risky as it might injure the organs, another disadvantage is that the CT or MRI data should contain this marker information, which means performing a CT or MRI after attaching markers to the patient. This is regarded as highly inconvenient. On the other hand, external tracking systems, both optical and electromagnetic, have their own disadvantages and are intrusive to the surgical environment. Optical tracking poses problems when there is no proper line of sight or good lighting conditions [21] and electromagnetic tracking has encountered problems when metallic objects are present in the operated area [22]. Apart from that, the above mentioned technologies have failed to provide a good approach to compensate the motion on both rigid and deformable organs without the use of markers or external tracking. Finally, these technologies have failed to provide a solution to use the projector to visualize information on the patients under surgical lights. There are commercially available pico projectors that have good illuminance and luminous flux [23] but none of them match the illuminance provided by the surgical light.

Our research therefore focuses on bridging these gaps between the existing technologies mentioned so far by developing a high illuminance hand-held projector system, which can accurately project information not only on rigid organs but also on deformable organs without the use of markers and external tracking systems. At the same time to compensate for various quick movements of the device by the surgeon, motion compensation algorithms are planned to be developed which will improve the robustness of the device. To build a high illuminance hand projector system various requirements have to be considered and this paper aims to provide and analyze those requirements that are needed for building such a special hand-held projector with respect to our application, i.e. the open liver surgery.

\section{OPTICAL REQUIREMENTS FOR AN AUGMENTED REALITY PROJECTOR}

This section describes the optical requirements for designing an augmented reality projector for surgical interventions. A workflow of the process is shown in Figure 1. The workflow constitutes of analyzing a special task at first. Here our main task is to project important anatomical information such as vessel structures, tumors etc. on the liver. For the surgeons to visualize the projected information on the liver under the surgical lights, various factors that enhance the visibility contrast of the projected information are analyzed. From this analysis the optical requirements to build an augmented reality projector are derived. The contents of the workflow are discussed in the subsections below. 


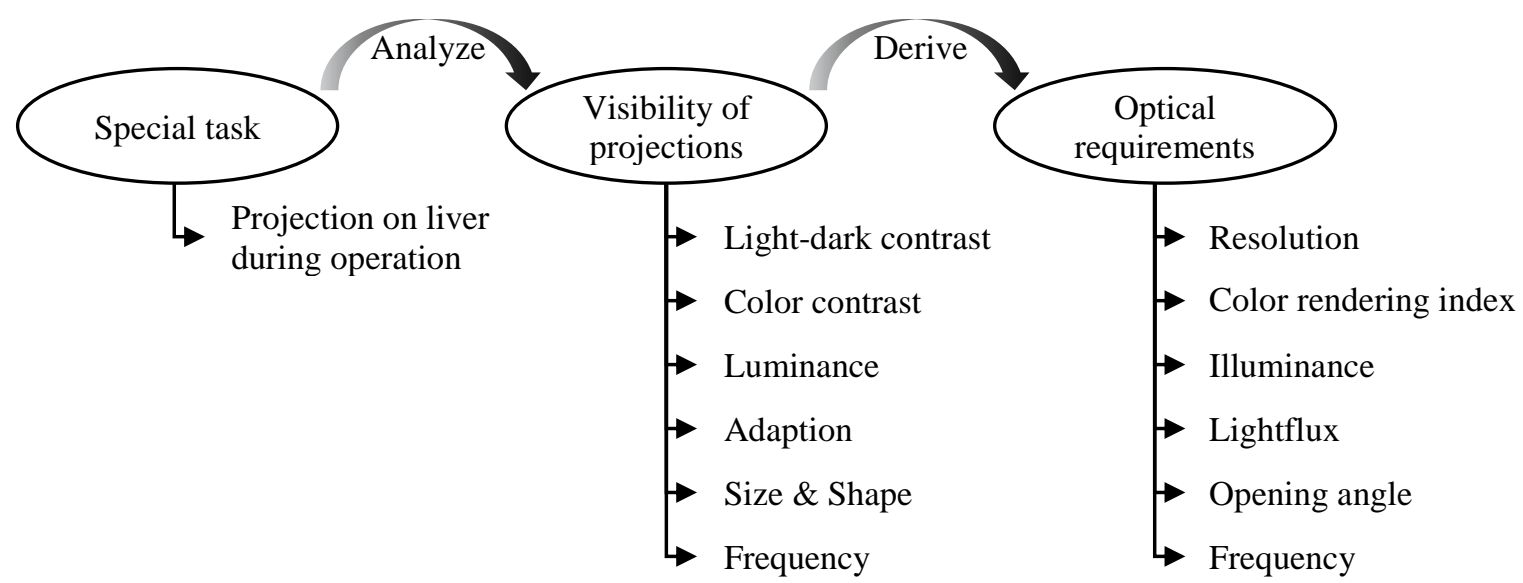

Figure 1: Workflow to design a special projector

\subsection{Analysis of the specific application}

For the visibility of objects Hartmann defines five necessary conditions [24]. They are:

- The object must have a minimum contrast (color or luminance contrast).

- The object must have a minimum size.

- The object or the environment must have a minimum luminance.

- The eye must be optimally adapted to the field luminance.

- The object must be visible for a certain amount of time to be recognizable.

The visibility of the projection depends strongly on the contrast of the projected structures [25,26]. Contrast is distinguished in light-dark and in color contrast. The light-dark contrast corresponds to the physical contrast $\mathrm{K}$ and can be determined using the measured luminance.

$L_{\text {Obj }}:$ Luminance of the object

$$
K=\frac{\left(L_{\mathrm{Obj}}-L_{\mathrm{U}}\right)}{L_{\mathrm{U}}} \cdot 100 \%
$$

$L_{\mathrm{U}}$ : Field luminance

With $L_{\mathrm{P}}$ as luminance of projector the luminance of the object can be calculated to

$$
L_{\mathrm{Obj}}=L_{\mathrm{U}}+L_{\mathrm{P}}
$$

And therefore:

$$
K=\frac{L_{P}}{L_{\mathrm{U}}} \cdot 100 \%
$$

The high illuminance of surgical lamps has a strong influence on the field luminance. In a preliminary experiment, a luminance of about $1,000 \mathrm{~cd} / \mathrm{m}^{2}$ was measured, which was generated by a surgical light with about 50,000 lx.

The size of the projected structures is in the millimeter range. Together with the distance of the operator's eyes from the illuminated organ, the minimum size of the projected structure is about a few angular minutes (arcmin). To show the influence of the minimum object size on the necessary contrast, the following estimation is performed for a minimum object width of $1 \mathrm{~mm}$ and $3 \mathrm{~mm}$, respectively. With a maximum projection distance of $600 \mathrm{~mm}$ (arm length), we get a viewing angle of $17^{\prime}$ for $3 \mathrm{~mm}$ and 5,7' for $1 \mathrm{~mm}$. 
Figure 2 shows the relationship between contrast, object size and luminance. For visibility, a contrast of at least $2 \%$ is necessary at a luminance of about $1,000 \mathrm{~cd} / \mathrm{m}^{2}$ and an object size of $17^{\prime}$ and a contrast of at least $5 \%$ at 5,7 . In order to increase the visibility much higher contrast values should be generated with the projection system.

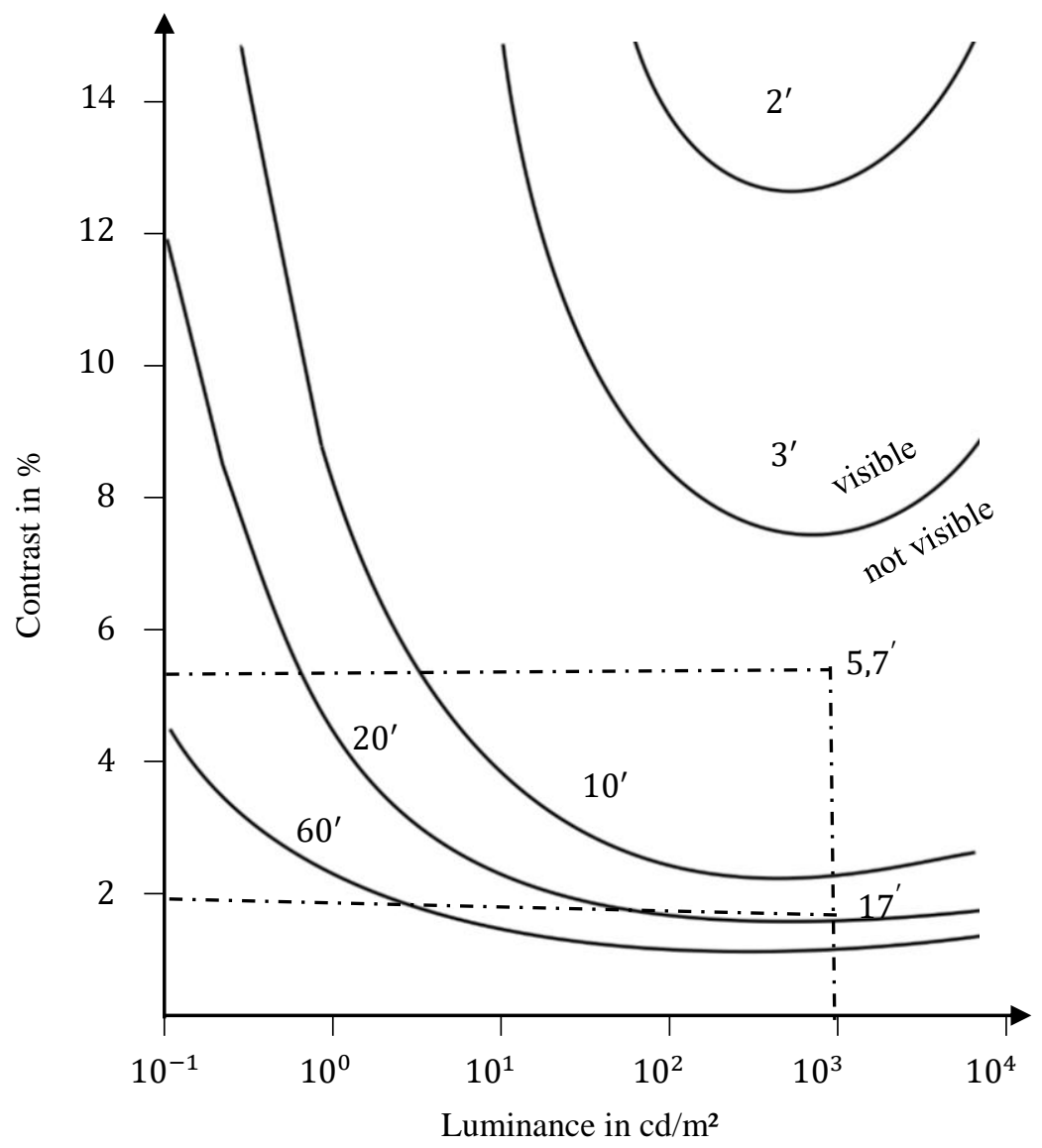

Figure 2: Correlation between contrast, size ( $\operatorname{arcmin})$ and luminance of an object (data from [27])

The size of the projection surface is chosen so that a projection on the complete size of the liver as the largest organ in the human body is possible. Verma et al. published the linear dimensions of the liver of 116 individuals as a result of MRI scans [28]. In the top view (mid hepatic point, craniocaudad) the size in the subject collective is $7.1-18.3 \mathrm{~cm}$. The size of the projection surface is chosen based on it and should be at least a square of $25 \mathrm{~cm}$ edge length. A circular or oval projection surface is also possible.

The influence of the frequency of projections on the visibility is divided into two effects. The flicker fusion threshold describes the frequency at which just no flicker occurs in a fast image sequence. In photopic vision, this flicker fusion frequency is dependent on the luminance. The maximum is about $90 \mathrm{~Hz}$ and thus represents the most critical case for which the system is to be designed [29].

The visibility of the projection can be increased with a dynamic projection. For the design of the blinking frequency, e.g. DIN EN 842-2009 can be consulted, in which a frequency of $2 \mathrm{~Hz}$ to $3 \mathrm{~Hz}$ with the same on and off intervals is proposed for flashing lights. In order to examine how a flashing projection can positively influence the visibility, a corresponding user study is carried out. Intermittent light stimuli with a frequency of $5 \mathrm{~Hz}$ to $15 \mathrm{~Hz}$ activate the nerve cells in the human eye particularly strongly and can therefore cause seizures for people with epilepsy [29]. The flashing frequency of the projections should therefore be below $5 \mathrm{~Hz}$. 


\subsection{Derivation of the optical requirements}

In this section, based on the previous analysis, optical requirements for an OR (operation room) projector are derived.

\section{Resolution}

A possibility to dimension the projector is based on the assumption that the smallest structures to be projected have a diameter of about $3 \mathrm{~mm}$. To be able to show radii, it is required that the projector can project pixels with $1 \mathrm{~mm}$ edge length. The size of the projection surface results in a minimum required resolution of 250 x 250 pixels.

The visual acuity of a healthy person in the fovea centralis is about 1 / minute angle [29]. At a working distance of about $600 \mathrm{~mm}$, the eye can resolve structures down to a minimum of $0.17 \mathrm{~mm}$. Based on the dimensions of a human liver; a projection size of at least $250 \mathrm{~mm} \times 250 \mathrm{~mm}$ is required. This results in a maximum useful resolution of $1470 \times 1470$ pixels.

\section{Color rendering index (CRI)}

The CRI value describes how natural the color of an illuminated object looks like. For the sunlight or blackbody (e.g., incandescent), a CRI of 100 is defined. In order to see the actual color of tissue, a high CRI light source is necessary. For OR lamps, IEC 60601-2-41 regulates that the CRI value for Surgical Lights (OR lamps) must be at least 85.

As part of this paper, the projector is used to produce a so-called positive contrast. This means that information can be read by locally increased luminance. Since the surgical light is switched on at the same time, writing is done with projecting a luminance or color difference on an already illuminated surface. In this setup, it is useful for the projector to reach a CRI value of 85 to achieve the required minimum color rendering index even in areas illuminated by both light sources. With a correspondingly high CRI value of the OR lamp, the value of the projector may be less than 85 , as long as both light sources together achieve sufficient color reproduction.

If, on the other hand, the projector is only used to illuminate fine structures without contributing to the general illumination of the tissue, its CRI value is of subordinate importance. Alternatively, the generation of a negative contrast is conceivable with very powerful projectors. Here, the surgical lights are partially or completely replaced by projectors, which now illuminate the entire organ. In this way the structures that should be displayed are visualized in black. Since the projector is used instead of a surgical light in this case, it must meet the requirements for surgical lights also in terms of the color rendering index. Of particular importance, is the faithful reproduction of the red spectral range of visible light to allow the surgeon, for example, to estimate the oxygen content of the blood. The red spectrum is evaluated with the indicator R9, which is not part of the conventional CRI value and which is therefore considered separately for surgical lights.

\section{Illuminance}

In Section 2.1 it was deduced that the luminance contrast on the organ should be at least 5\%. Assuming that a typical surgical lamp generates an illuminance of approximately 100,000 lx, the OR projector should be able to generate at least 5,000 lx. This relationship between the illuminance of the projector and the illuminance of the OR lamp applies only if both light sources have a similar emission spectrum.

\section{Light flux}

Together with the required illuminance of 5,000 $\mathrm{lx}$ and the projection area of $250 \mathrm{~mm} \times 250 \mathrm{~mm}$, this results in a minimum luminous flux of the projector of about $312.5 \mathrm{~lm}$. Taking the maximum angle of $45.2^{\circ}$ into account this value is increased to $1,250 \mathrm{~lm}$. A significantly increased luminous flux is strongly recommended to increase the visibility of projection on an inhomogeneous surface like the liver.

\section{Opening Angle}

The selected working distance of $600 \mathrm{~mm}$ results in a minimum horizontal and vertical opening angle of the projector of $23.5^{\circ}$. For a shorter working distance the illuminated area becomes smaller and for a higher distance the resulting contrast decreases. For a maximum distance of $300 \mathrm{~mm}$ an angle of $45.2^{\circ}$ is required. Since the projection is possible with distances the maximum angle of $45.2^{\circ}$ is chosen.

\section{Frequency}

The refresh rate of the projector must be well above the flicker fusion frequency of $90 \mathrm{~Hz}$. 


\section{MATERIALS AND METHODS}

This section describes the methodology that is involved in performing the user study to design an AR projector. The user study was conducted in an experimental OR. The details about the experimental setup and methods used in the study are discussed below in detail.

The experimental setup consists of a high illuminance DLP projector (Panasonic PT-DZ110XE) to project vessel structures of the desired sizes on a cow's liver under the surgical lights. This projector has an illuminance of approximately 107,000 lux at distance of $60 \mathrm{~cm}$. The illuminance of the surgical lights (2x Polaris 560 DC) manufactured by Dräger was measured to be approximately 100,000 lx at a distance of one meter. A LMK 5 luminance camera, having a resolution of 1,380 x 1,030 pixels, developed by Technoteam Bildverarbeitung GmbH is used to measure the luminance contrast of the projected information. Parts of a fresh cow's liver used, mimicked the properties of human liver in terms of reflections and occlusions. This made the study for the users even more interesting. Figure 3 a) shows the picture the entire measurement setup used in these user study experiments and Figure 3 b) shows the picture of the cow's liver used in the user study.

The user study is divided into three parts. The idea behind each part is to analyze the visibility contrast of the projected vessel structure under the surgical lights by varying different properties of the projection. The study is conducted with eleven users, out of which three are surgeons. A C++ GUI based application using OpenCV, Visualization Toolkit (VTK) and QT libraries is used with an intension to project the vessel structure accurately at a same position on the liver for all users. The projector and a stereo camera system are calibrated with respect to each other prior to projecting the vessel structures which increases the accuracy of the projection. The projector screen is rescaled to a resolution of $800 \mathrm{x}$ 600 pixels and then calibrated. The projected image entirely covers the cow's liver. At this resolution, the image size is approximately $40 \mathrm{~cm}$ x $30 \mathrm{~cm}$. This constitutes to approximately 50 pixels per inch (PPI) and 2 pixels per mm.

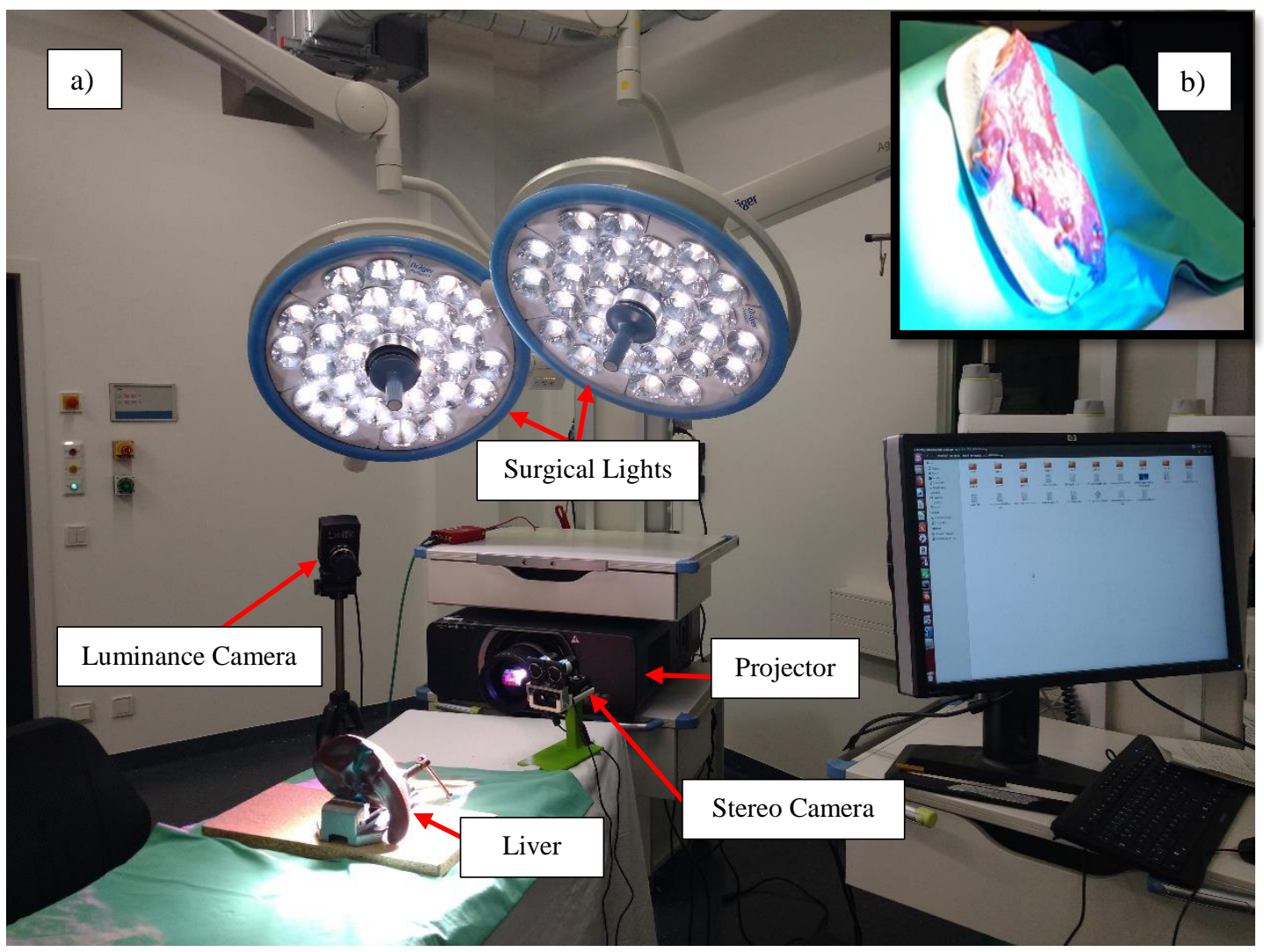

Figure 3 a): Experimental setup for the user study. b) Picture of the cow's liver used for the user study 


\subsection{User Study 1: Evaluation of color combinations}

The first part of the user study deals with the visibility of the projected information by varying the background and foreground (vessel structure) color combination of the projected information. The goal is to choose the best color combination for rendering the projected information in this scenario. A previous user evaluation study performed by our group (in print) filtered out the best five color combinations from a series of 15 color combinations based on the visibility contrast on both a pig's liver and an artificial liver. These five color combinations are given in Table 2 .

For this study the projection (a vessel structure with a line width of $3 \mathrm{~mm}$ ) is static. The users evaluate the visibility contrast of color combination based on scale ranging from 0 to 3 (where 3 denotes the best visibility and 0 the worst visibility). The best color combination would be kept as constant for the user studies 2 and 3 for each test person.

Table 2: Background and foreground color combinations

\begin{tabular}{|c|c|}
\hline Background Color & Foreground Color \\
\hline Black & White \\
\hline White & Black \\
\hline White & Red \\
\hline White & Blue \\
\hline Blue & Yellow \\
\hline
\end{tabular}

\subsection{User Study 2: Evaluation of projection frequency}

In the second user study, the visibility contrast of the projected information is analyzed while varying the frequency of the projected information. The projected vessel structure (foreground information) is switched on and off for different pre-decided intervals of time while the background color and the vessel size remain constant. The color combination that the user preferred in the previous part of the user study is taken into account for this part. Apart from the pre-decided intervals of switching time, the user also has an option to choose individual on- and off-intervals. This study also evaluates the nervousness or the calmness of the user when it comes to dynamic projection via their feedback. In the end, the users had to provide a feedback about their preference of dynamic projection over static projection and whether the dynamic projection improves the visibility contrast under the surgical lights when compared to the static projection. Again the users evaluate the visibility contrast on the scale ranging from 0 to 3.

\subsection{User Study 3: Evaluation of vessel thickness}

In the third user study, the visibility contrast of the vessel structure is analyzed by keeping the color combination of the static projection constant while changing the vessel thickness. For the previous two user studies, a vessel thickness of $3 \mathrm{~mm}$ (6 pixels) is used. The purpose behind this study is to gradually decrease the thickness of the vessels to an extent where the surgeon or the user is no longer able to see the vessel structure properly with the surgical lights switched on. This gives us an idea about the minimum thickness of the vessel structure that can be shown to the surgeons during surgery. So the vessel thickness is varied from a maximum size of $3 \mathrm{~mm}$ to a minimum size of $0.5 \mathrm{~mm}$. OpenCV thin plate splines (TPS) and lens undistortion techniques are used to warp the projected image, so that a constant thickness of desired pixels could be maintained throughout the vessel structure while projecting it on a deformable organ like the liver. Before using these lens undistortion methods, surface acquisition of the liver is carried out through stereo reconstruction techniques.

\section{RESULTS}

In this section the results of all the three user studies are given. The first user study deals with the variation in background and foreground color of the projected information, the second study deals with the variation in projection 
frequency (dynamic projection) and the third study deals with variation in vessel thickness. In all our studies the same visibility evaluation scale is used: 3-very good, 2-good, 1-acceptable, 0-bad.

\subsection{User Study 1: Evaluation of color combinations}

From Figure 4, it is very clear that the blue background and yellow foreground was the most preferred color combination. Interestingly all the 11 users in the user study picked this combination over other color combinations. The overlaid upper and lower whiskers cover the maximum and the minimum value for each color combination. The " $x$ " in the center represents the mean of the visibility scale value for each color combination.

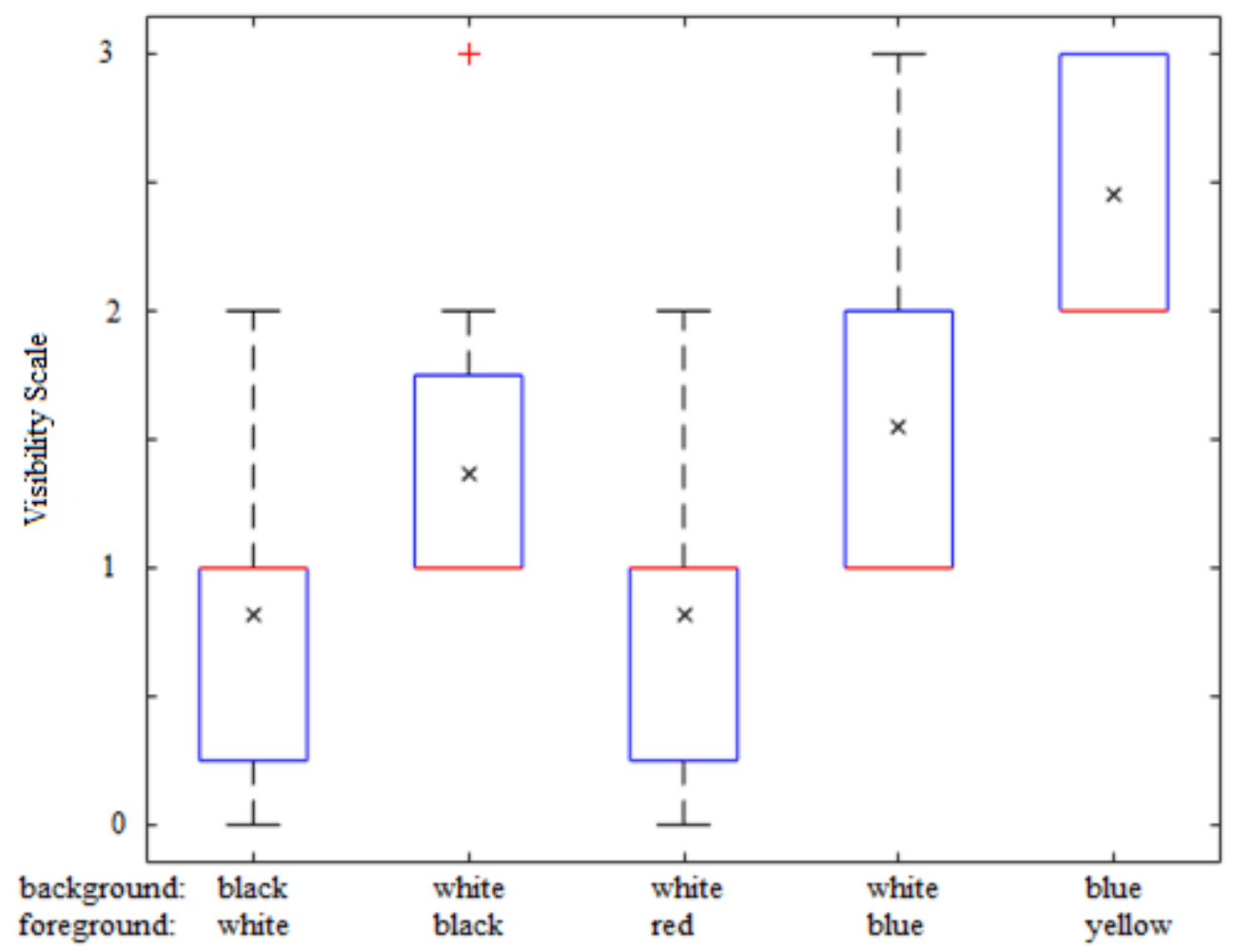

Figure 4: Results of User Study 1 (Evaluation of color combinations)

Figure 5 provides information about the frequency of ratings for each color combination based on the visibility scale. For example, there were 3 people who chose 0 as the visibility level for black background, white foreground and white background, red foreground respectively. From this data we can figure out that the blue background and yellow foreground was clearly the most preferred color combination as 6 users rated it a 2 and 5 users rated it a 3 in accordance to the visibility evaluation scale. 


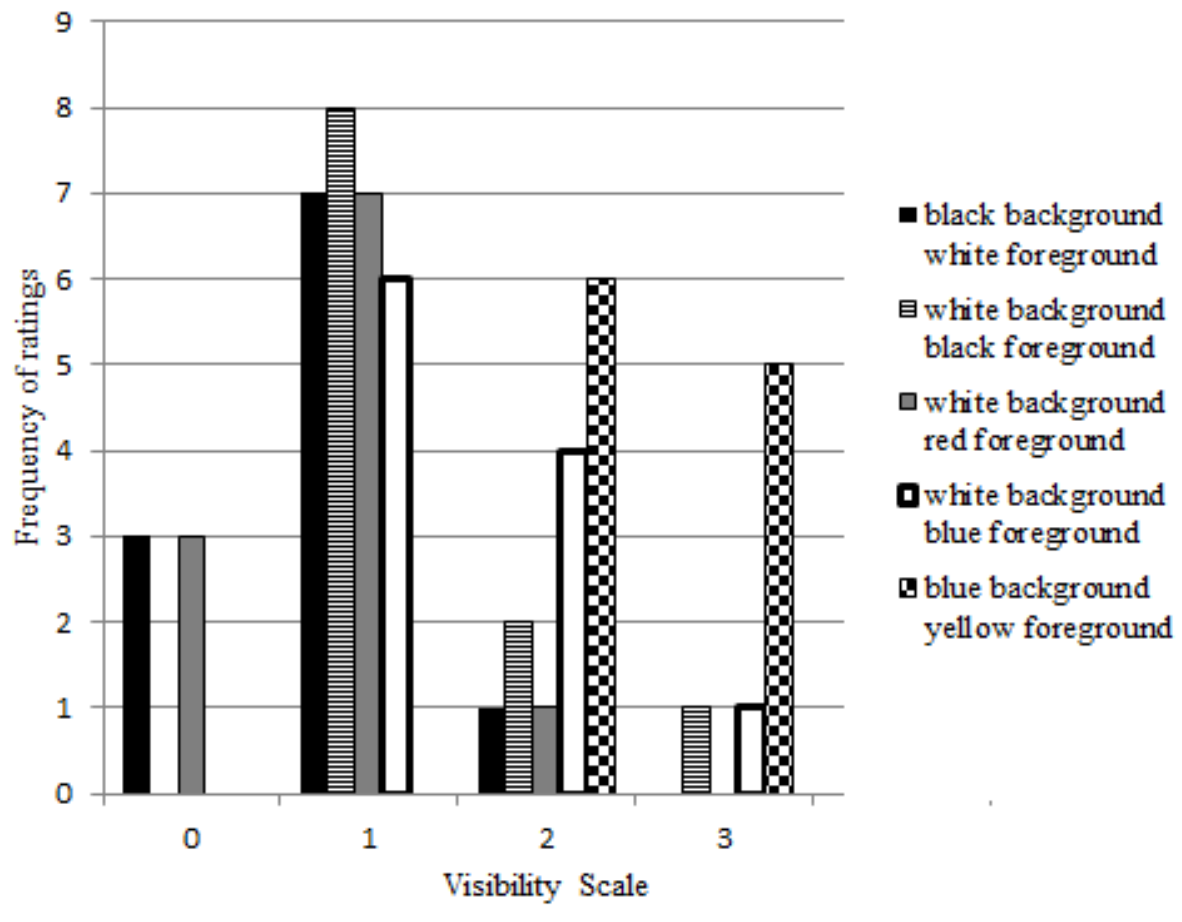

Figure 5: Frequency rating in terms of visibility scale (Evaluation of color combinations)

The Figure 6 shows the luminance image of the liver with projected structures on it. Caused by the inhomogeneous surface and specular reflections, the luminance of the liver ranges from about $1,300 \mathrm{~cd} / \mathrm{m}^{2}$ up to $18,000 \mathrm{~cd} / \mathrm{m}^{2}$. The mean value in the projection area is about $4,500 \mathrm{~cd} / \mathrm{m}^{2}$. Inside the black circle two projected horizontal vessel structures are barely visible. Due to the low physical contrast of the color projection with yellow foreground and blue background it is not reasonable to calculate a Weber contrast in this case. Nevertheless, the projected structure is clearly visible to the user's eye.

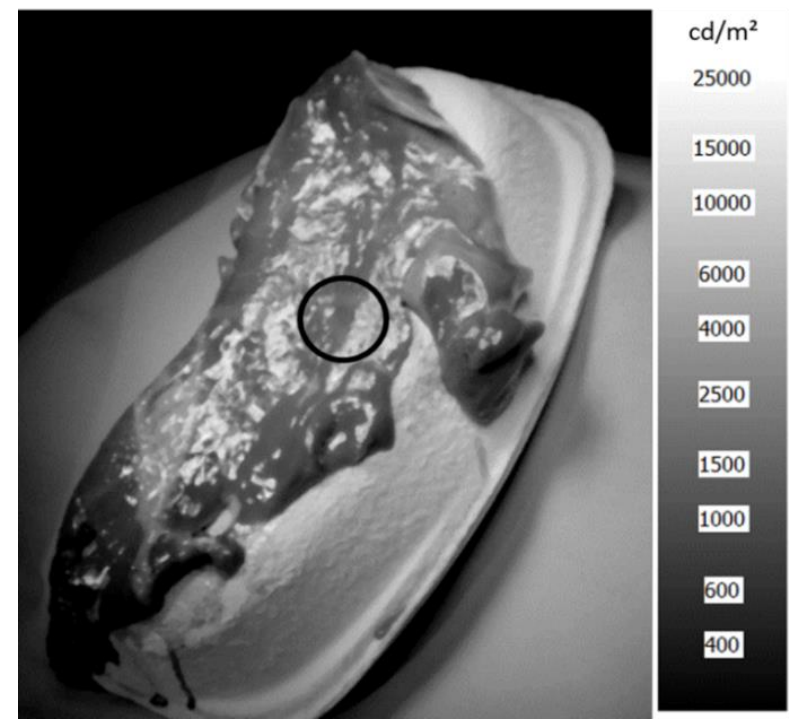

Figure 6: Luminance measurement of liver with projection (blue background, yellow foreground) 


\subsection{User Study 2: Evaluation of projection frequency}

The best color combination chosen by each user during the previous user study is taken into account for this part. The projected vessel thickness is constantly maintained at $3 \mathrm{~mm}$. All test persons make use of the yellow-blue projection for this and the next scenarios. Table 3 summarizes the results of this user study in the form of a statistical analysis. Since there is no significant preference for a switching frequency or on-off-ratio, a table with statistical analysis in terms of Mean, Standard Deviation (SD), Median, Maximum and Minimum values in terms of visibility scale is considered better for this user study.

Table 3: Statistical analysis for User Study 2 in terms of visibility evaluation scale values

\begin{tabular}{|c|c|c|c|c|}
\hline Groups & $\begin{array}{c}\text { on-time 250 ms } \\
\text { off-time 100 ms }\end{array}$ & $\begin{array}{c}\text { on-time 500 ms } \\
\text { off-time 200 ms }\end{array}$ & $\begin{array}{c}\text { on-time 1000 ms } \\
\text { off-time 200 } \mathbf{~ m s}\end{array}$ & $\begin{array}{c}\text { on-time 500 ms } \\
\text { off-time 500 ms }\end{array}$ \\
\hline Mean \pm SD & $1.54 \pm 0.93$ & $1.63 \pm 0.67$ & $1.63 \pm 0.92$ & $1.81 \pm 0.87$ \\
\hline Median & 1 & 2 & 2 & 2 \\
\hline Min & 0 & 0 & 0 & 0 \\
\hline Max & 3 & 2 & 3 & 3 \\
\hline
\end{tabular}

\subsection{User Study 3: Evaluation of vessel thickness}

In this user study, the visibility contrast of the vessel structure is evaluated by varying it's thickness in pixels. The best color combination chosen by the user in the first user study is kept constant. The projection is static. The results of this study in which 1 pixel stands for a line width of approximately $0.5 \mathrm{~mm}$ are given in Table 4 . Here again we prefer a statistical analysis table instead of box plots. Here the thickest structure is the best in terms of visibility for several users. Six of eleven test persons rate the slightly thinner structure $(2 \mathrm{~mm}$ instead of $3 \mathrm{~mm})$ as comparable.

Table 4: Statistical analysis for User Study 3 in terms of visibility evaluation scale values

\begin{tabular}{|c|c|c|c|c|}
\hline \multirow[t]{2}{*}{ Groups } & \multicolumn{4}{|c|}{ Vessel Size } \\
\hline & $3 \mathrm{~mm}$ & $2 \mathrm{~mm}$ & $1 \mathrm{~mm}$ & $0.5 \mathrm{~mm}$ \\
\hline Mean \pm SD & $\begin{array}{c}2.90 \pm \\
0.30\end{array}$ & $2.45 \pm 0.52$ & $1.45 \pm 0.52$ & 0 \\
\hline Median & 3 & 2 & 1 & 0 \\
\hline Min & 2 & 2 & 1 & 0 \\
\hline Max & 3 & 3 & 2 & 0 \\
\hline
\end{tabular}

\section{DISCUSSION AND CONCLUSION}

The study discussed in this paper is based on the results of eleven test persons including three surgeons. The test persons are between the age of 29 and 51. The mean age of the test persons is 34 . Including the surgeons, there were five people who have a good experience in open surgeries. Considering all this, the results give important hints for the requirements of an augmented reality projection system for surgical interventions but do not take into consideration all important factors like the different age categories of the users, limitations like color blindness or the spectrum of the light source completely. Nevertheless, the most obvious result is that the projection of yellow vessel structures on a blue background is beneficial for all test persons. Although the Weber contrast is reduced in comparison to white structures on a black 
background the additional color difference enhances the visibility of the structure projected on the cow's liver in the in vitro experiments.

While some test persons like a static projection of the information others prefer a dynamic on- and off-switching of the image. Seven out of the eleven users preferred dynamic projection over static projection. However, the surgeons did not approve of the dynamic projection. They felt that the static projection was better and made them less nervous. This part of the study gives very subjective results with no clear preferences for a switching frequency or on-off-ratio. Since the dynamic projection helps to differentiate the projected information from the real structure on the organ's surface, in addition to the static mode, a dynamic projection mode should be implemented in the system offering user-specific onoff-intervals.

The required size of the projected structure depends on the contrast which the projection system can generate on the organ. Therefore, the test persons rated the thickest structure (line width $\sim 3 \mathrm{~mm}$ ) as the best visible one. For a thinner structure with a width of $\sim 2 \mathrm{~mm}$ the visibility was still sufficient. In order to give the surgeon precise information about the position of important structures the projected lines should be as thin as possible. However, there is a point below which the surgeons will not be able to see the vessel structure clearly anymore. In our case, the users determined that the structure with a thickness below $1 \mathrm{~mm}$ was not clearly visible. Hence the vessel structures having a thickness below 1 $\mathrm{mm}$ would not be shown to the surgeons during surgery.

The users were also asked to provide a feedback about the flickering of the projected image. At a refresh rate of $60 \mathrm{~Hz}$, none of the users were able to see any flickering. However, in the previous user study, there was one of the users who could witness image flickering at $60 \mathrm{~Hz}$. In Section 2.1, it is already stated that the maximum threshold till which a person can notice image flickering is $90 \mathrm{~Hz}$ [30]. Since flickering of the image tends to disturb the surgeons during the surgery, it is advisable to design a projector with a refresh rate more than $90 \mathrm{~Hz}$.

This user study is a preliminary step towards providing the requirements for building a hand-held projector system for augmented reality applications in surgery. The significance of these results has to be proven definitely with a more realistic setup in future. In the next step, a concept or a prototype for a handheld projection system would be set up. Since the yellow-blue projection yields a very good visibility a two-color projection system might be a good approach in order to reduce the system size in comparison to conventional three-color RGB devices. Such a system can e.g. be based on two laser sources and a micromirror (MEMS) $[25,30]$. One important advantage of the yellow-blue color space in comparison to other two-color spaces is the possibility to generate white light by overlaying both colors.

\section{ACKNOWLEDGEMENT}

The authors would like to thank the workgroup CAS-hno for providing us the experimental OR at Niedersächsisches Zentrum für Biomedizintechnik, Implantatforschung und Entwicklung (NIFE), to perform our user study. The project, which this paper is a part of, is funded by the Lower Saxony Ministry for Science and Culture as part of the Tailored Light scholarship program.

\section{CONFLICT OF INTEREST}

The authors declare that there was no conflict of interest in relation to the content of the manuscript. 


\section{References}

[1] Seattle-malpractice-lawyers., "Shocking Surgical Error Statistics," 16 February 2017, http://www.seattlemalpracticelawyers.com/blog/2017/02/shocking-surgical-error-statistics/ (01 April 2017).

[2] Jennifer Warner,. "Thousands of Mistakes Made in Surgery Every Year,” 26 December 2012, https://www.webmd.com/a-to-z-guides/news/20121220/thousands-mistakes-surgery\#1 (01 April 2017).

[3] Kumar, S. and Steinebach, M., "Eliminating US hospital medical errors. International Journal of Health Care Quality Assurance," 21(5), 444-471(2008).

[4] Gavaghan, K.A., Peterhans, M., Oliveira-Santos, T. and Weber, S., "A portable image overlay projection device for computer-aided open liver surgery," IEEE transactions on biomedical engineering, 58(6), 1855-1864 (2011).

[5] Gavaghan, K.A., Anderegg, S., Peterhans, M., Oliveira-Santos, T. and Weber, S., "Augmented reality image overlay projection for image guided open liver ablation of metastatic liver cancer," Workshop on Augmented Environments for Computer-Assisted Interventions, 36-46 (2011).

[6] Gavaghan, K., Oliveira Dos Santos, T., Peterhans, M., Reyes, M., Kim, H. and Weber, S., "Portable image overlay projection for the visualization of surgical planning and navigation data," (2011).

[7] Sugimoto, M., Yasuda, H., Koda, K., Suzuki, M., Yamazaki, M., Tezuka, T., Kosugi, C., Higuchi, R., Watayo, Y., Yagawa, Y. and Uemura, S., "Image overlay navigation by markerless surface registration in gastrointestinal, hepatobiliary and pancreatic surgery," Journal of hepato-biliary-pancreatic sciences, 17(5), 629-636 (2010).

[8] Tardif, J.P., Roy, S. and Meunier, J., "Projector-based augmented reality in surgery without calibration," Proc of the 25th Annual International Conference of the IEEE, 1, 548-551 (2003).

[9] Edgcumbe, P., Pratt, P., Yang, G.Z., Nguan, C. and Rohling, R., "Pico lantern: a pick-up projector for augmented reality in laparoscopic surgery," International Conference on Medical Image Computing and Computer-Assisted Intervention, 432-439 (2014).

[10] Ni, T., Karlson, A.K. and Wigdor, D., "AnatOnMe: facilitating doctor-patient communication using a projection-based handheld device," Proc. of the SIGCHI Conference on Human Factors in Computing Systems, 3333-3342 (2011).

[11] Besharati Tabrizi, L. and Mahvash, M., "Augmented reality-guided neurosurgery: accuracy and intraoperative application of an image projection technique," Journal of neurosurgery, 123(1), 206-211 (2015).

[12] Kobler, JP., Hussong, A., and Ortmaier, T., "Mini-projector based augmented reality for medical applications," CURAC Tagungsband, 115-118, (2010).

[13] Krempien, R., Hoppe, H., Kahrs, L., Daeuber, S., Schorr, O., Eggers, G., Bischof, M., Munter, M.W., Debus, J. and Harms, W., "Projector-based augmented reality for intuitive intraoperative guidance in image-guided 3D interstitial brachytherapy," International Journal of Radiation Oncology Biology Physics, 70(3), 944-952 (2008).

[14] Strobl, K.H., Mair, E., Bodenmüller, T., Kielhöfer, S., Sepp, W., Suppa, M., Burschka, D. and Hirzinger, G., "The self-referenced DLR 3D-modeler," Proc. Intelligent Robots and Systems, IROS, IEEE/RSJ International Conference, 21-28 (2009).

[15] Schwier, A., Konietschke, R., Bodenmüller, T., Ende, T., Kielhöfer, S. and Hirzinger, G., "VR-Map: A New Device for Patient Registration and Optimal Robot Positioning," Proc. of CURAC, 111-114 (2010).

[16] Shi, C., Becker, B.C. and Riviere, C.N., "Inexpensive monocular pico-projector-based augmented reality display for surgical microscope," Proc. Computer-Based Medical Systems (CBMS), 25th International Symposium, 1-6 (2012).

[17] Glossop, N., Wedlake, C., Moore, J., Peters, T. and Wang, Z., "Laser projection augmented reality system for computer assisted surgery," International Conference on Medical Image Computing and Computer-Assisted Intervention, 239-246 (2003). 
[18] Watts, I., Boulanger, P. and Kawchuk, G., "ProjectDR: augmented reality system for displaying medical images directly onto a patient," Proc. of the 23rd ACM Symposium on Virtual Reality Software and Technology, 70 (2017).

[19] Chae, M.P., Ganhewa, D., Hunter-Smith, D.J. and Rozen, W.M., "Direct augmented reality computed tomographic angiography technique (ARC): an innovation in preoperative imaging," European Journal of Plastic Surgery, 1-6 (2018).

[20] Wu, J.R., Wang, M.L., Liu, K.C., Hu, M.H. and Lee, P.Y., "Real-time advanced spinal surgery via visible patient model and augmented reality system," Computer methods and programs in biomedicine, 113(3), 869881 (2014).

[21] Koivukangas, T., Katisko, J.P. and Koivukangas, J.P., "Technical accuracy of optical and the electromagnetic tracking systems," Springerplus, 2(1), 90 (2013).

[22] Schicho, K., Figl, M., Donat, M., Birkfellner, W., Seemann, R., Wagner, A., Bergmann, H. and Ewers, R., "Stability of miniature electromagnetic tracking systems," Physics in Medicine \& Biology, 50(9), 2089 (2005).

[23] Ayaz, S.M. and Kim, M.Y., "Multiview registration-based handheld 3D profiling system using visual navigation and structured light," International Journal of Optomechatronics, 11(1), 1-14 (2017).

[24] Hartmann, E., [Beleuchtung und Sehen am Arbeitsplatz], W. Goldmann, (1970).

[25] Kloppenburg, G., "Scannende Laser-Projektionseinheit für die Fahrzeugfrontbeleuchtung," TEWISS - Technik und Wissen GmbH Verlag, (2017).

[26] Rizvi, S., Ley, P.P., Knöchelmann, M. and Lachmayer, R., "Road safety enhancement: an investigation on the visibility of on-road image projections using DMD-based pixel light systems," Proc. SPIE 10554, 105541Q (2018).

[27] Bubb, H., Vollrath, M., Reinprecht, K., Mayer, E. and Körber, M., [Der Mensch als Fahrer], Automobilergonomie, Springer Fachmedien Wiesbaden, 67-162 (2015).

[28] Verma, S.K., McClure, K., Parker, L., Mitchell, D.G., Verma, M. and Bergin, D., "Simple linear measurements of the normal liver: interobserver agreement and correlation with hepatic volume on MRI," Clinical radiology, 65(4), 315-318 (2010).

[29] Schmidt, R.F., Lang, F. and Heckmann, M. eds., [Physiologie des Menschen: Mit Pathophysiologie], SpringerVerlag, (2011).

[30] Kloppenburg, G., Wolf, A. and Lachmayer, R., "High-resolution vehicle headlamps: technologies and scanning prototype," Advanced Optical Technologies, 5(2), 147-155 (2016). 